\title{
Semantic Query and Reasoning System based on Domain Ontology
}

\author{
SONG Wan $\mathrm{Li}^{1,2 \text {, a }}$, NI Li Xian ${ }^{3, b}$ \\ ${ }^{1}$ Nanjing Communications Institute of Technology, Nanjing, 211188, \\ China \\ ${ }^{2}$ School of Computer and Information, Hohai University, Nanjing, 211100, \\ China \\ ${ }^{3}$ The People's Bank of China Lianyungang Central Sub-branch, Jiangsu \\ Lianyungang, 222002, China \\ aswlhhu@hhu.edu.cn, ${ }^{b}$ nilixian@hhu.edu.cn
}

\begin{abstract}
To solve the problems such as isolation of information, poor sharing and reusability, difficulty of finding resources and providing personalized information service, a solution method of ontology-based query and reasoning was introduced. According to the method a function module and working process of retrieval, query and reasoning were designed. A corresponding system was implemented by using Jena and SPARQL. The implementation and experimental results indicated that the method was feasible and effective. It is concluded that this system can provide organic organization, sharing and reusing of information content, and personalized information service.
\end{abstract}

Keywords: domain ontology; semantic query; semantic reasoning; Jena.

\section{Introduction}

In the traditional information system, information was stored in a fixed hierarchical database or information base. Information structure mainly relied on representation based on hypertext. Most of the information was isolated relatively. Sharing and reusing of information was relatively poor. "Trek" phenomenon occurred frequently in finding resources. Personalized service demand could not 
be met. How to improve the information system has become a common concern to researchers.

Conventional information retrieval technology based on keywords can not meet the demand of people for information query. Semantic web [2] technology is the most promising approach to solve this problem [1]. As a conceptual modeling tool which can describe the information system in semantic layer and information layer, ontology is located in the core position in the hierarchy of semantic Web [3] The semantic web technology based on ontology is applied in many fields. Literature [4] studied the ontology based semantic retrieval technology, and established a semantic retrieval prototype on the basis of corn planting ontology. Literature [5] studied and designed a semantic retrieval system based on domain ontology , designed and built university domain ontology and implemented a semantic retrieval function. The literature [6] introduced the basic concept of ontology theory, implemented a healthy information model system of lung cancer based on ontology, and gave the main framework of the system. Literature [7] presented the framework architecture of an E-learning course, and then created course ontology. Above literatures introduced ontology into the corresponding fields, but were not deep enough. Some remained in the stage of theoretical model. Others just implemented simple prototypes. All of them did not provide semantic reasoning functions.

In this paper, we design domain ontology of courses. Then we construct a Semantic Query and Reasoning System based on Domain Ontology (SQRS-DO), and we give the design and implementation of this system and the analysis of running results, verify the effectiveness and feasibility of semantic query and reasoning based on course ontology.

\section{System Design and Realization}

Architecture and Main Process. The architecture and process flow of SQRS-DO is illustrated in Fig. 1. The main process flow is as follows: 
(1) User selects the corresponding retrieval terms in the retrieval interface, and submits them to the retrieval condition generator. Generator generates specific retrieval conditions according to

(2) retrieval items, runs semantic retrieval function. The corresponding results will be retrieved from the ontology information base by using Jena APIs and shown to the user.

(2) User inputs or selects a corresponding query fragment in the query interface, submits to the SPARQL statement assembler. The assembler adds necessary information to the submitted fragment to generate a complete SPARQL query statement, and then calls semantic query execution to query corresponding results from the information base by executing the complete SPARQL statement.

(3) User selects or input the rule information in reasoning rule interface, submits it to the reasoning rule loader. The loader loads the reasoning rules calls semantic reasoning engine to derive additional assertions from existing information base by using Jena reasoning engine.

(4) User calls information base exporting engine in reasoning interface. The exporting engine makes new information stored in memory persistent to the database by using the database API of Jena. 


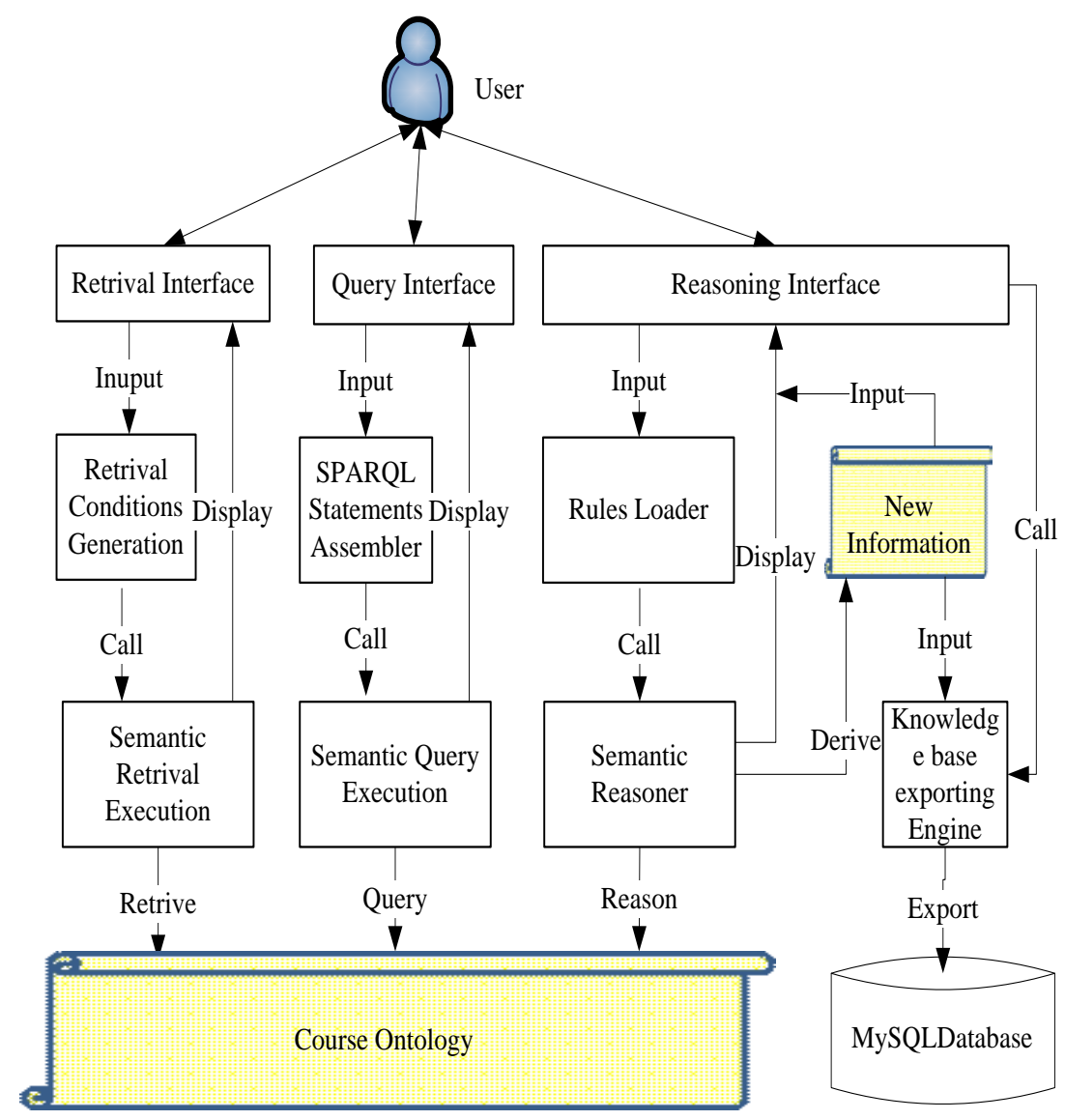

Figure 1. Architecture and process flow of SQRS-DO

System Implementation. SQRS-DO is mainly composed of four modules, including classification retrieval, semantic query and semantic reasoning, information base exporting. All modules are independent of each other. They realize semantic query and reasoning tasks together based on course ontology. Classification retrieval relies on information structure and content. Semantic query is realized by using SPARQL [8]. Semantic reasoning is supported by using Jena's own reasoning mechanism. New information base is derived from the information system ontology and exported to various relational database by using the Jena RDF API. 
SQRS-DO follows the three layer architecture of MVC (Model-View-Controller), and is developed based on Java EE 6.0 platform and Apache Jena framework. It is a Web application being deployed in Apache Tomcat 7.0 server. The front-end of SQRS-DO is implemented with Extjs2.0, and the back-end is coded in the open-source frameworks of Struts2 and Spring3.

Domain Ontology Building. The domain ontology is designed and developed by using Protégé[13], which contains total 3 classes, including KnowledgePoint, Teacher and Category. This paper mainly takes the KnowledgePoint class as example. The properties of KnowledgePoint class are shown in table 1.

Table 1. Properties of KnowledgePoint class in domain ontology

\begin{tabular}{|c|c|c|c|c|}
\hline Property & Domain & Range & Type & Description \\
\hline precursor & KnowledgePoint & KnowledgePoint & $\begin{array}{c}\text { Object } \\
\text { property }\end{array}$ & $\begin{array}{c}\text { pre order } \\
\text { information } \\
\text { point }\end{array}$ \\
\hline parallel & KnowledgePoint & KnowledgePoint & $\begin{array}{c}\text { Object } \\
\text { property }\end{array}$ & $\begin{array}{c}\text { parallel } \\
\text { information } \\
\text { point }\end{array}$ \\
\hline isClassified & KnowledgePoint & Category & $\begin{array}{c}\text { Object } \\
\text { property }\end{array}$ & classification \\
\hline chargeTeacher & KnowledgePoint & Teacher & $\begin{array}{c}\text { Object } \\
\text { property }\end{array}$ & $\begin{array}{c}\text { teacher in } \\
\text { charge }\end{array}$ \\
\hline hasName & Thing & string & $\begin{array}{c}\text { Data } \\
\text { property }\end{array}$ & name \\
\hline informationDes & KnowledgePoint & string & $\begin{array}{c}\text { Data } \\
\text { property }\end{array}$ & $\begin{array}{c}\text { description } \\
\text { of } \\
\text { information } \\
\text { point }\end{array}$ \\
\hline hasURL & KnowledgePoint & string & $\begin{array}{c}\text { Data } \\
\text { property }\end{array}$ & URL \\
\hline createTime & KnowledgePoint & date & $\begin{array}{c}\text { Data } \\
\text { property }\end{array}$ & creating time \\
\hline applications & KnowledgePoint & string & $\begin{array}{c}\text { Data } \\
\text { property }\end{array}$ & application \\
\hline difficultLevel & KnowledgePoint & int & $\begin{array}{c}\text { Data } \\
\text { property }\end{array}$ & $\begin{array}{l}\text { degree of } \\
\text { difficulty }\end{array}$ \\
\hline
\end{tabular}




\section{Results and Analysis}

The knowledge points and their corresponding preorder knowledge points can be queried from the domain ontology. The corresponding SPARQL query statement is as follows:

PREFIX kld: <http://www.njci.edu.cn/swl/ontologies/information\#>

PREFIX rdf: <http://www.w3.org/1999/02/22-rdf-syntax-ns\#>

SELECT ?name, ?kp WHERE \{ ?name rdf:type kld:KnowledgePoint.

OPTIONAL \{ ?name kld:precursor ?kp. \} \}

The query results are consistent with the instances of the domain ontology. The implementation indicates that our approach is feasible using semantic query in the information system. The isolated information is organized together, and can be shared and reused.

According to the actual needs of the system, we formulate corresponding reasoning rules and stored in rules documents. The rules are shown as following.

(1) [successor: (?b kld:precursor ?a),notEqual(?b, ?a)->(?a kld:successor ?b)]

(2) [isEasy:(?c rdf:type kld:KnowledgePoint),(?c kld:difficultLevel ?d), greaterThan(?d,0),lessThan(?d,5)->(?c kld:isEasy ?d)]

(3) [isHard:(?e rdf:type kld:KnowledgePoint),(?e kld:difficultLevel ?f), greaterThan(?f,5)->(?e kld:isHard ?f)]

We will illustrate the rules defined in the rule file. Rule 1, if you want to learn knowledge point $\mathrm{A}$ and $\mathrm{B}$ and $\mathrm{A}$ are not the same knowledge point, you must first learn knowledge point $\mathrm{B}$, namely "precursor" and "successor" are inverse functions. Rule 2, if the difficulty degree of knowledge point is between $0-5$, this knowledge is defined as easy and students can grasp better. Rule 3, if the difficulty degree of knowledge point is greater than 5 , the knowledge point is defined as hard, and students must study harder to grasp it. The rule file is dynamically loaded by Jena framework when the system is running.

The result data can be successfully generated by after loading the rules in SQRS-DO. The reasoning results are consistent with the data of actual ontology 
instances. It verifies that the results are reliable, and we can achieve different results by different reasoning rule specification.

\section{Summary}

We apply the semantic query and reasoning technology to information system to solve the problems, such as information points isolation, poor sharing and reusability, "Trek" learning, difficulty to find learning resources, not providing personalized services. The semantic retrieval, query and reasoning functions are designed and implemented based on information system ontology. The implementation and experimental results indicate that the proposed approach is feasible and effective and further intensify the ontology technology research in the field of information system. Our future work mainly focuses on increasing the degree of correlation between the information system ontology and the learning resources and enhancing the retrieval method and to recommend the right learning resource to the right student.

\section{Acknowledgements}

This work was financially supported by the Jiangsu Natural Science Foundation (BK20141420), the Research Foundation of Nanjing Communications Institute of Technology (No. JY1108, No. JY1403) and the Education Science Twelfth Five-Year Plan Project of Jiangsu Province (No. B-b/2013/03/04).

\section{References}

[1] L. X. NI, T. WU. "Semantic Query and reasoning System Based on Domain Ontology”, Computer and Modernization, Vol. 225 (2014), p. 74-78.

[2] T. Berners-Lee, J. Hendler, O.Lassila. "The Semantic Web", Scientific American, Vol. 284, No. 5 (2001), p.34-43.

[3] Z. Q. GAO, Y. PAN, L. MA . "Semantic Principles and its Applications", Machinery Industry Press, Beijing(2009). 
[4] L. L. ZHANG. "The Research of Semantic Retrival Based on Domain Ontolgoy”, master degree thesis of Jilin University, Changchun (2009).

[5] C. G. SUN. "Research of Semantic Retrival Technology Based on the Universiy Domain Ontolgoy”, master degree thesis of North University of China, Taiyuan (2012).

[6] G. M. YUAN, B. FAN, X. L. CHEN, T. H. CHENG. "Study on Application of Computer Ontology Theory in Lung Cancer”, Mocrocomputer Applications, Vol. 30, No. 5 (2014), p.44-47.

[7] H. LI, G. Z. YANG. “Course development of e-Learning based on Ontology”, Computer Engineering and Design, Vol. 31, No. 4 (2010), p.881-884.

[8] SPARQL Language for

http://www.w3c.org/TR/2008/REC-rdf-sparql-query-20080115/ 